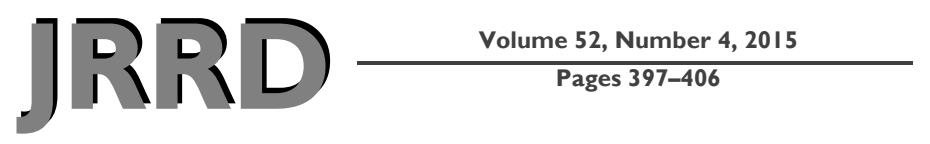

\title{
Surface electrical stimulation to evoke referred sensation
}

\author{
Johanna C. Forst; Derek C. Blok; Julia P. Slopsema; John M. Boss; Lane A. Heyboer; Carson M. Tobias; \\ Katharine H. Polasek, PhD* \\ Department of Engineering, Hope College, Holland, MI
}

\begin{abstract}
Surface electrical stimulation (SES) is being investigated as a noninvasive method to evoke natural sensations distal to electrode location. This may improve treatment for phantom limb pain as well as provide an alternative method to deliver sensory feedback. The median and/or ulnar nerves of 35 subjects were stimulated at the elbow using surface electrodes. Strength-duration curves of hand sensation were found for each subject. All subjects experienced sensation in their hand, which was mostly described as a paresthesia-like sensation. The rheobase and chronaxie values were found to be lower for the median nerve than the ulnar nerve, with no significant difference between sexes. Repeated sessions with the same subject resulted in sufficient variability to suggest that recalculating the strength-duration curve for each electrode placement is necessary. Most of the recruitment curves in this study were generated with 28 to 36 data points. To quickly reproduce these curves with limited increase in error, we recommend 10 data points. Future studies will focus on obtaining different sensations using SES with the strength-duration curve defining the threshold of the effective parameter space.
\end{abstract}

Key words: artificial sensation, electrical stimulation, feedback, paresthesia, phantom limb pain, referred sensation, sensation, sensory feedback, strength-duration curve, surface electrodes.

\section{INTRODUCTION}

Surface electrical stimulation (SES) is a noninvasive method to interface with the nervous system. When used to target the nerve trunk, SES can activate muscles and/or sensations in locations other than the point of stimulation. For example, stimulation of the median nerve at the elbow could produce muscle activation and/or sensation in the hand. Sensations obtained using SES are often described as tingling or prickling (paresthesias). In an individual with an amputation, these paresthesias can be felt in the missing limb and are being investigated as a treatment for phantom limb pain [1]. An improvement in the SES technique to produce natural sensations such as touch may improve the treatment for phantom limb pain as well as provide a noninvasive method to deliver sensory feedback.

There are multiple methods under investigation to restore sensation to prosthetic users. Sensory substitution methods use electrocutaneous stimulation [2-3] or vibrotactile stimulation [4] to translate information such as grip force to the residual limb of the user. Haptic methods employ mechanisms such as force applicators [5] or pneumatic bladders [6] to provide a more realistic sensation of touch or pressure, but still on the residual limb. To obtain sensation that is referred to the missing limb, force applicators have been used on skin surgically reinnervated by nerves of the hand [7]. Electrical stimulation of the residual nerves has also been used to elicit referred sensation in the missing limb. The first implanted system consisted of an electrode placed near the median nerve

\footnotetext{
Abbreviations: PEST $=$ Parameter Estimation by Sequential Testing, RMS = root mean square, $\mathrm{SES}=$ surface electrical stimulation.

*Address all correspondence to Katharine H. Polasek, PhD; Assistant Professor of Engineering, Hope College, 223F Vanderwerf, 27 Graves PI, Holland, MI 49423; 616395-7079; fax: 616-395-7123. Email: polasek@hope.edu http://dx.doi.org/10.1682/JRRD.2014.05.0128
} 
and successfully provided sensations as if they were coming from the hand [8]. Improvements in technology have made an implanted system more feasible, and several groups have been re-evaluating the use of implanted nerve stimulation to communicate sensory information. Using wires inserted into the nerve, tactile-like sensations have been produced that change in magnitude and location based on the stimulation [9-11]. In addition, Tan et al. used nerve cuff electrodes to produce sensations at different locations in the hand through extraneural stimulation and found that nonstandard waveforms can elicit touch-like sensations without paresthesias [12]. The goal of this research is to develop a noninvasive method to elicit natural, referred sensation. This article reports preliminary work focused on defining the parameter space.

Threshold values for electrical stimulation can be described using a stimulus amplitude and duration relationship called a strength-duration curve. Prior studies investigating the strength-duration curve for SES of sensory fibers used compound action potentials to quantify the response [13-14] rather than reported referred sensation. Establishing this baseline relationship for referred sensation was an important first step to looking at the ability of electrical stimulation to produce natural sensations.

In this article, we report the testing of three hypotheses. First, SES at the elbow can produce referred sensation in the hand without causing discomfort in other locations. Second, the strength-duration curve can be found using reported sensation as a measure of threshold rather than a physiological measurement. Third, it is possible to produce a natural sensation using SES.

\section{METHODS}

\section{Experimental Setup}

Subjects between the ages of 18 and 65 were recruited from the Hope College campus community. The protocol was approved by the Hope College Human Subject Review Board, and all subjects gave written informed consent. Each study session lasted from 45 to $90 \mathrm{~min}$, and subjects were compensated for their time. To look at variability between sessions, we tested 10 of the subjects at a second session, at least a week later than their first visit.

Prior to their scheduled session, subjects were instructed to drink plenty of fluids to reduce their skin impedance. They were also given a bottle of water to drink during the trial. After giving consent, subjects were instructed to wash their left elbow and then were seated in an upright chair with their left arm extended on a padded table. No hair removal was performed. Rubbing alcohol was used to clean the skin before applying the electrodes over the median or ulnar nerve (Figure 1). For median nerve stimulation, electrodes were placed over the biceps tendon with the cathode placed proximal to the anode. For ulnar nerve stimulation, electrodes were placed over the groove between the olecranon of the ulna and the medial epicondyle of the humerus on the back of the elbow (where the ulnar nerve is most superficial). A photograph was taken of the electrodes to document their location and an elastic band was wrapped around the arm to assist electrode adhesion. Subjects' hands were hidden from view prior to stimulation to prevent a disparity between what was being felt and seen.

Stimulation was supplied via voltage-controlled, charge-balanced, biphasic, nonsymmetric pulses. Pulses were nonsymmetric in that the anodic phase was set to a maximum value of $4 \mathrm{~V}$, with a width as needed to balance the charge [15-16]. This was done to prevent activation under the anode. The stimulation waveforms were created in MATLAB (The MathWorks, Inc; Natick, Massachusetts) and delivered using a National Instruments USB DAQ (NI USB-6229; Austin, Texas) and an isolated biostimulator (model A13-75, Coulbourn Instruments; Pittsburgh, Pennsylvania, or BIOPAC STIMSOLA; Goleta, California). Adhesive electrodes were cut to $30 \mathrm{~mm}$ by

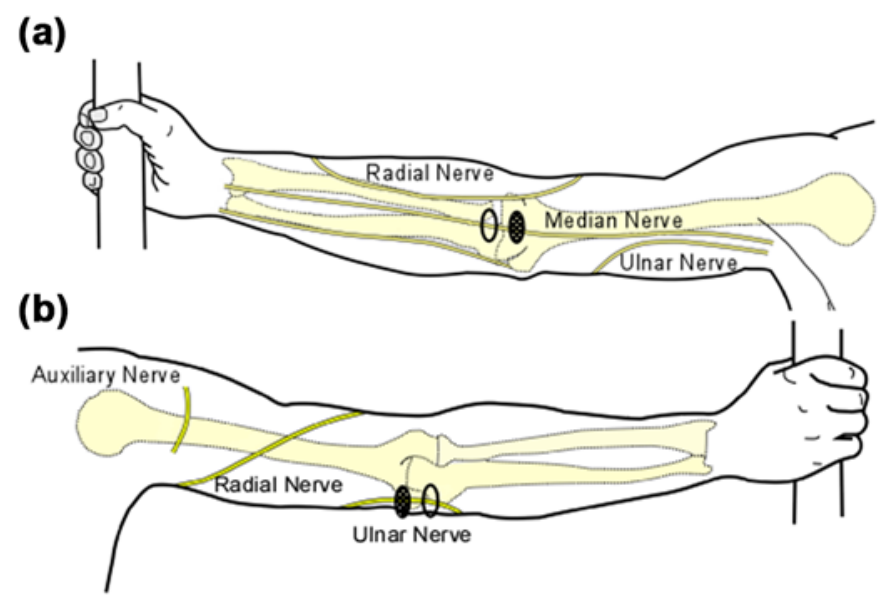

Figure 1.

Electrode location for targeting (a) median nerve and (b) ulnar nerve. Filled ovals are active electrodes and open ovals are return electrodes. Figure modified from Grey $\mathrm{H}$. Anatomy of the human body. Philadelphia (PA): Lea \& Febiger; 1918. 
17 mm (ValuTrode, Axelgaard Manufacturing; Fallbrook, California). Data were recorded in MATLAB throughout testing based on participant feedback.

Testing began by ramping the voltage amplitude in increments of $0.5 \mathrm{~V}$ at a constant $500 \mu$ s pulse duration and $50 \mathrm{~Hz}$ frequency to allow the subject to become accustomed to the stimulus and determine approximate hand sensation threshold values. Ramping continued until the subject indicated his or her maximum comfort level, which was not reached again throughout the remainder of the study. The subjects were then instructed to adjust their arm position until maximum distal sensation was achieved while minimizing muscle activation and forearm sensation. Once an acceptable arm position was found, subjects were asked to refrain from arm and shoulder movements for the remainder of the test and a photograph was taken to document their position.

\section{Data Collection}

An adaptive psychophysical procedure, Parameter Estimation by Sequential Testing (PEST) [17-18], was used to determine threshold values for sensation in the hand using a range of pulse duration and amplitude values. PEST is a method of threshold detection that was developed to obtain a more accurate estimation of sensory data and remove some of the variability and bias that occur when collecting data using subject reporting. The procedure consisted of starting with a subthreshold stimulus and increasing until a hand sensation was reported and then decreasing until the sensation disappeared. This was continued with a decreasing step size until the step size was within a specified tolerance (Figure 2). The tolerance ranged from 0.5 to 2.5 percent of the threshold value.

Two protocols were used to determine the strengthduration curve over the course of testing. The original protocol was modified after the first set of subjects to increase the number of data points at lower pulse duration values. In Protocol 1, PEST was used to determine the threshold at 3 constant pulse durations (100, 500, and $1,000 \mu \mathrm{s})$ and 4 constant pulse amplitudes ( 7 total threshold points), with 4 responses at each point (28 total data points). In Protocol 2, the number of durations was increased to $5(50,100,300,500$, and $1,000 \mu \mathrm{s})$ for 9 threshold points, with 4 responses at each point (36 total data points). In each case, the pulse amplitude voltages were chosen to span the range from threshold to maximum comfort that was estimated during initial ramping. Repeated stimulation values were presented in a quasirandom order.

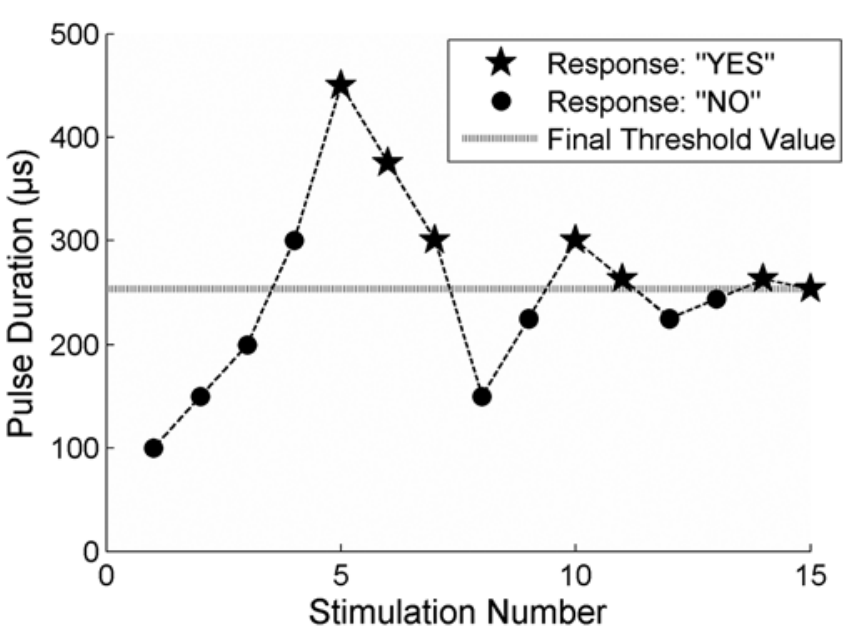

Figure 2.

Example of using PEST (Parameter Estimation by Sequential Testing) to determine threshold from subject 2 with pulse amplitude of $29.5 \mathrm{~V}$. Stimulation duration was varied ( $y$-axis) at each stimulation number ( $x$-axis), and subjects reported "YES" if they felt sensation in their hand or "NO" if they did not. Horizontal line depicts final threshold value.

\section{Strength-Duration Curve}

A strength-duration threshold curve along with its rheobase and chronaxie values was constructed from the threshold points. The rheobase $\left(V_{\mathrm{Rh}}\right)$ is defined as the lowest amplitude required for nerve activation at infinite pulse durations. The chronaxie $\left(\tau_{C}\right)$ is the corresponding pulse duration at twice the rheobase. A linear relationship between the voltage equivalent of charge (the product of threshold voltage, $V_{\text {th }}$, and threshold pulse duration, $t$ ) and pulse duration was used to estimate the rheobase voltage and the chronaxie time using Equation 1 [19]. The fitted strength-duration curve was generated using the rheobase voltage, the chronaxie time, and Equation 2. This method has been found to produce the best fit for strength-duration data $[13,20]$ and is illustrated in Figure 3.

$$
\begin{aligned}
& V_{\mathrm{th}} \times t=V_{\mathrm{Rh}}\left(t+\tau_{C}\right) . \\
& V_{\mathrm{th}}=V_{\mathrm{Rh}}\left(1+{ }^{\tau_{C}} / t\right) .
\end{aligned}
$$


(a)

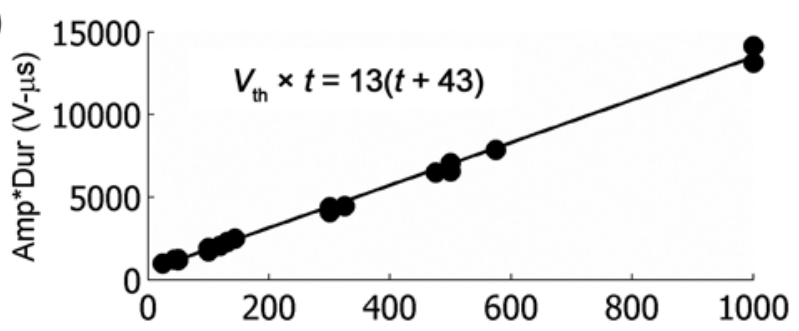

(b)

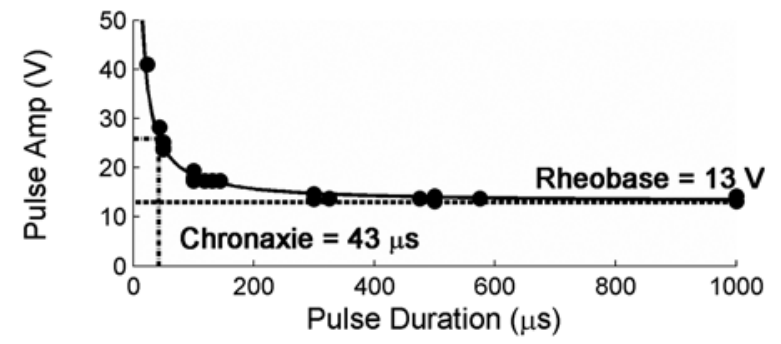

Figure 3.

Example strength-duration curve calculation from subject 20 .

(a) Product of pulse amplitude (Amp) and duration (Dur) was plotted against pulse duration. This linear relationship was used to calculate rheobase amplitude and chronaxie time using Equation 1. (b) Resultant strength-duration curve.

\section{Data Analysis}

The chronaxie and rheobase values were compared using a two-tailed Student $t$-test between the following: median and ulnar nerves, male and female subjects for each nerve, and different sessions for each repeated subject. Data from Protocol 2 were also analyzed to quantify the effect of the protocol change.

The curves in this study were obtained using 7 or 9 different threshold points, with 4 responses at each point for a total of 28 or 36 data points. In future experiments, it will be desirable to quickly estimate this threshold curve to define the effective parameter space prior to collecting more detailed sensation information. The previously collected data were used to quantify the effect of additional data points on the accuracy of the threshold curve. Strength-duration curves were calculated for each subject using from 2 to 7 threshold points and 1 to 4 responses at each point. The root mean square (RMS) error was used to quantify how well each curve, calculated with a subset of the data, fit all of the data points. To determine the least number of data points to sufficiently reproduce the strength-duration curve, we used a repeated-measures analysis of variance to produce pair- wise comparisons between the error at each subset of points and the error from using all data points.

\section{Current- Versus Voltage-Controlled Stimulation}

Voltage-controlled stimulation is preferential to current-controlled stimulation when using adhesive surface electrodes. A loss of adhesion would increase the impedance of the electrode-skin interface. In voltage-controlled stimulation, this would decrease the injected current, which is not desirable but would not cause a risk to research subjects. A loss of adhesion when using currentcontrolled stimulation would produce increased current density and potentially cause skin burns [21]. For this reason, voltage-controlled stimulation was chosen for use in this project.

In order to compare these data with previously reported results, we performed a supplemental trial on the median nerve using current-controlled stimulation. Strength-duration curves were found using the reduced number of points suggested to decrease collection time. Five threshold points were found with two responses at each point using PEST as described previously (10 total data points). The 5 points consisted of 2 constant pulse durations (100 and $500 \mu \mathrm{s})$ and 3 pulse amplitudes to span the range between threshold and maximum comfort level.

\section{RESULTS}

In all 46 testing sessions with 35 different subjects, distinguishable hand sensation was obtained from SES of the median or ulnar nerve at the elbow. The actual sensations varied from tingling to itchiness to pressing and were consistent with sensory innervation maps [22]. Hand and arm position varied between subjects but was maintained in a consistent position throughout testing of each individual subject. The main study consisted of 36 sessions and 25 different subjects, with 10 subjects participating in the comparison of repeated trials. Ten additional subjects were recruited to participate in the current-controlled stimulation study. Each study included an equal number of subjects of each sex; subjects ranged in age from 18 to $42 \mathrm{yr}$. A detailed breakdown of subjects by substudy is shown in Table 1 .

\section{Strength-Duration Curve}

Strength-duration curves for both the median and ulnar nerve are shown in Figure 4. The rheobase voltage 
Table 1.

Detailed breakdown of subjects in each substudy.

\begin{tabular}{lccc}
\hline \multicolumn{1}{c}{ Variable } & Main Study & Repetitions & CC vs VC* \\
\hline Median Nerve (Subject No.) & $1-10,20-22$ & $1,3,9-10$ & $26-35$ \\
Ulnar Nerve (Subject No.) & $5,11-19,23-25$ & $11-12,15-17,19$ & - \\
Mean Age (yr) & 24 & 25 & 19 \\
Left Handed (No.) & 1 & 0 & 1 \\
${ }^{*}$ "Cur
\end{tabular}

*Current-controlled (CC) versus voltage-controlled (VC) stimulation.

ranged from 6.8 to $26.7 \mathrm{~V}$ for the median nerve and from 14.8 to $32.7 \mathrm{~V}$ for the ulnar nerve. The chronaxie time ranged from 5.8 to $112.5 \mu$ s for the median nerve and from 4.1 to $63.1 \mu$ s for the ulnar nerve. Average values with standard deviations are shown in Table 2. No significant differences between the two protocols were found. Significant differences were found between the two nerves for both the rheobase voltage $(p<0.001)$ and the chronaxie time $(p<0.05)$. The average rheobase and chronaxie values were observed to be lower for female subjects than for males, but the differences were not significant $(p>0.05)$. There was also a relationship between the circumference of the arm and the rheobase voltage for the median nerve ( $r=0.3$; for the ulnar nerve, $r=-0.01)$, but this was not a significant correlation.

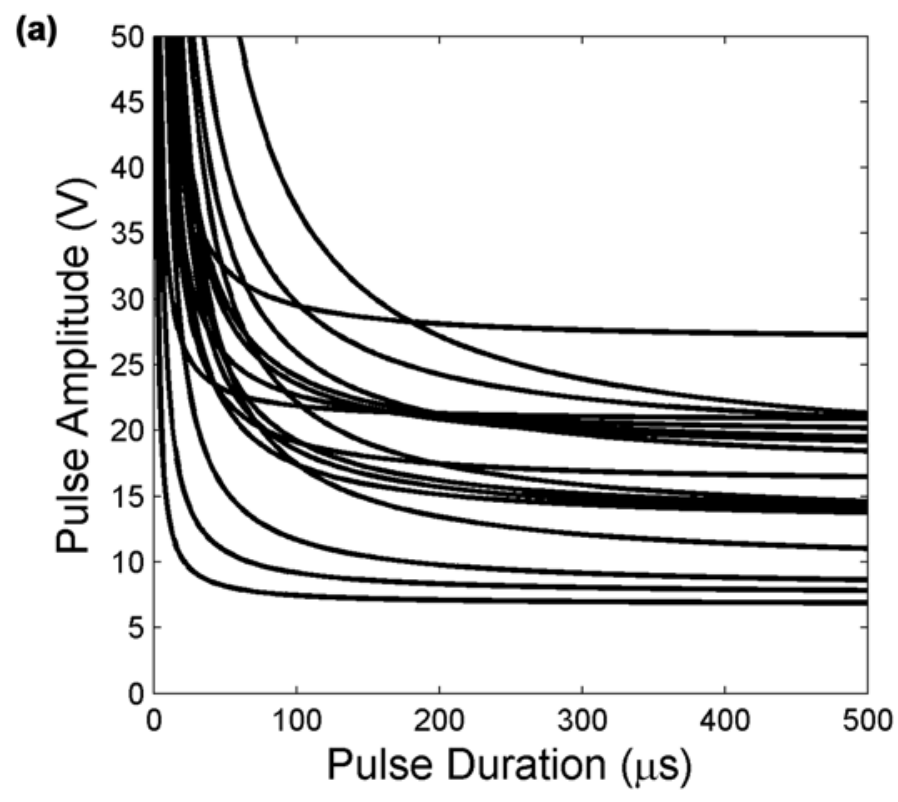

Figure 4.

Strength-duration curves from (a) median and (b) ulnar nerves.

\section{Variability in Strength-Duration Between Sessions}

Ten subjects participated in a repeated trial, four on the median nerve and six on the ulnar nerve (Figure 5). The percent difference between sessions for the rheobase voltage ranged from 5.3 to 36.2 percent for the median nerve and 1.7 to 66.7 percent for the ulnar nerve. The chronaxie time had a larger variability, with percent differences ranging from 3 to 137 percent.

\section{Efficient Calculation of Strength-Duration Curve}

The RMS error for each combination of number of threshold points and number of repeated responses is shown in Figure 6. As expected, the error for each number of responses decreased with the addition of threshold points. The error also decreased for each number of points with additional responses. For 2 responses per

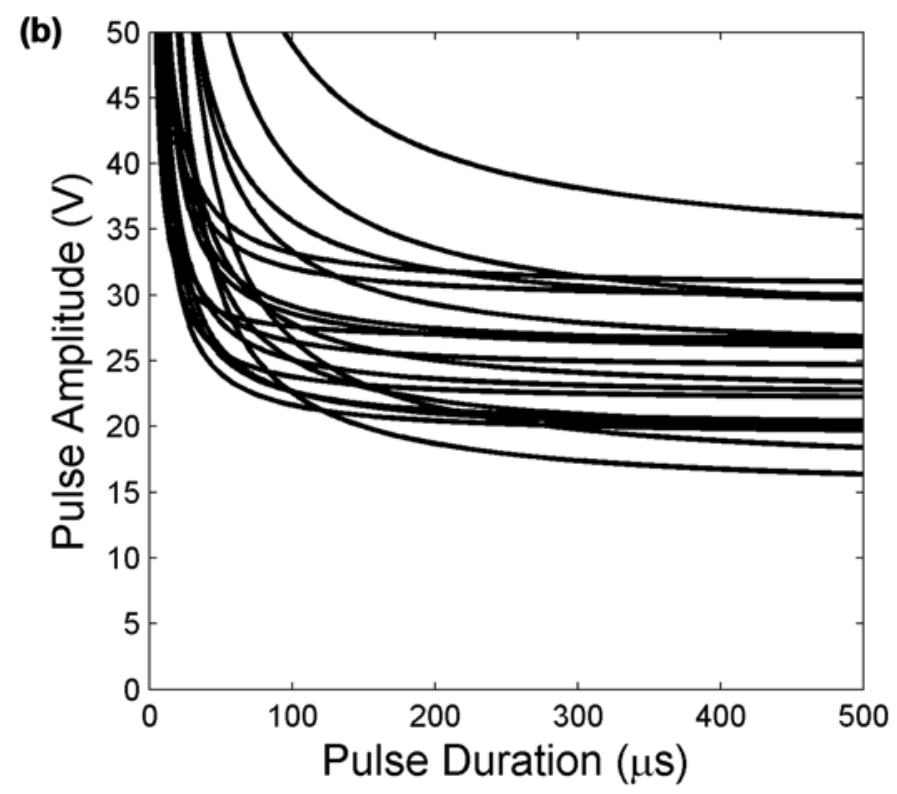


Table 2.

Average strength-duration curve properties.

\begin{tabular}{cccc}
\hline Variable & $\begin{array}{c}\text { Rheobase } \\
(\mathbf{V})\end{array}$ & $\begin{array}{c}\text { Chronaxie } \\
(\boldsymbol{\mu s})\end{array}$ & Number \\
\hline Median Nerve & $15.0 \pm 5.4$ & $41.5 \pm 29.1$ & 17 \\
Protocol 1 & $14.9 \pm 6.0$ & $42.6 \pm 30.7$ & 12 \\
Protocol 2 & $15.2 \pm 4.1$ & $38.9 \pm 28.0$ & 5 \\
Ulnar Nerve & $23.7 \pm 4.9$ & $23.6 \pm 17.7$ & 19 \\
Protocol 1 & $23.4 \pm 5.0$ & $25.7 \pm 19.4$ & 14 \\
Protocol 2 & $24.7 \pm 5.1$ & $17.7 \pm 10.8$ & 5 \\
\hline \hline
\end{tabular}

point, 5 or more threshold points resulted in error not significantly different than using all collected data points (10 or more total points). For 3 responses per point, 4 threshold points were needed, and for 4 responses per point, 3 threshold points were needed (12 total points for each case). There was more variability in the single response data so they were not included in the analysis. These results suggest that 10 data points ( 2 responses at each of 5 threshold points) will reduce the time required by 72 percent while not significantly increasing the error.

\section{Current- Versus Voltage-Controlled Stimulation}

Ten subjects participated in a supplemental experiment to compare current- versus voltage-controlled stimulation. The average rheobase current was $1.3 \pm 0.7 \mathrm{~mA}$. The average chronaxie time was $628 \pm 300 \mu$ s. Sensa- tions obtained were similar to those reported previously from voltage-controlled stimulation.

\section{DISCUSSION}

Hand sensation was obtained using SES of the median and ulnar nerve at the elbow in all subjects. Natural sensations were reported, but the vast majority of subjects experienced paresthesia-like sensations. In the past, strength-duration curves have been used to characterize neuromotor activity and nerve excitability, but here have been shown to also apply to referred sensation. There was sufficient variability between different sessions with the same subject to necessitate recalculating the strengthduration curve at each session, but it was found that 5 points with 2 responses at each point would sufficiently represent the curve. Finally, no difference was found between sensations reported using voltage-controlled versus current-controlled stimulation.

Data were found to support all three hypotheses. Strength-duration curves and hand sensation were found for all subjects. Natural sensations were reported, but rarely. Sensory information from mechanical receptors in the skin is coded using both frequency and timing of neuronal firing. For example, some receptors fire only at the beginning and end of a sensory event such as a touch. (a)

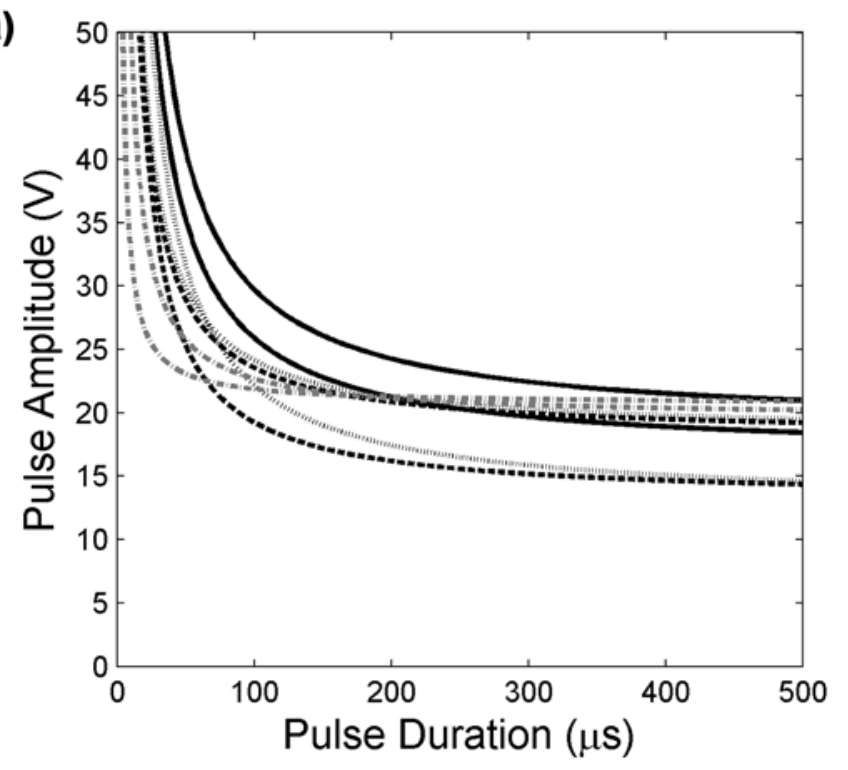

(b)

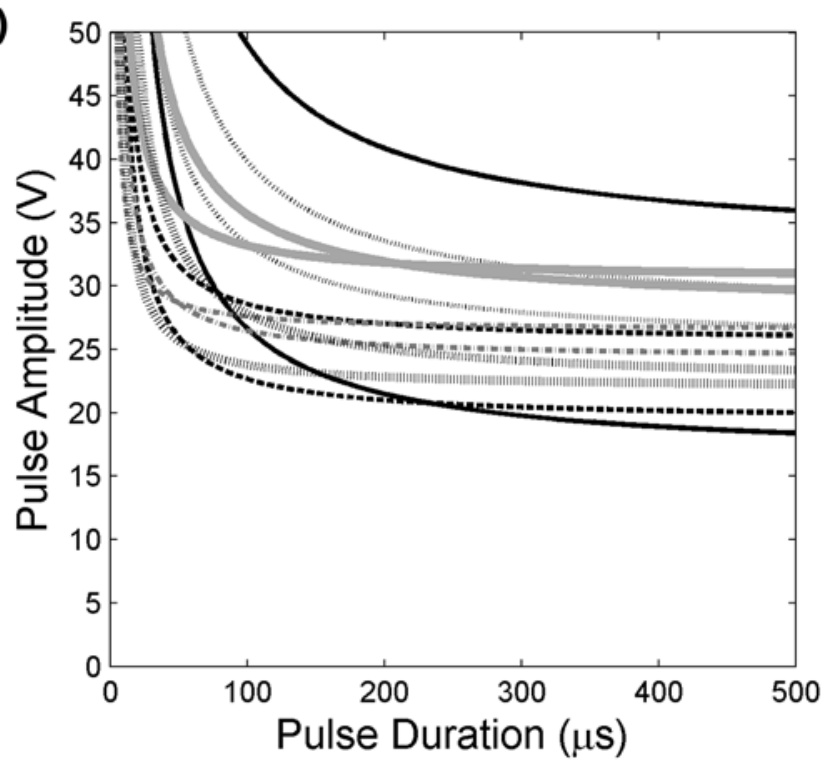

Figure 5.

Repeated trials from (a) median and (b) ulnar nerves. Curves from same subject are depicted using same line type. 


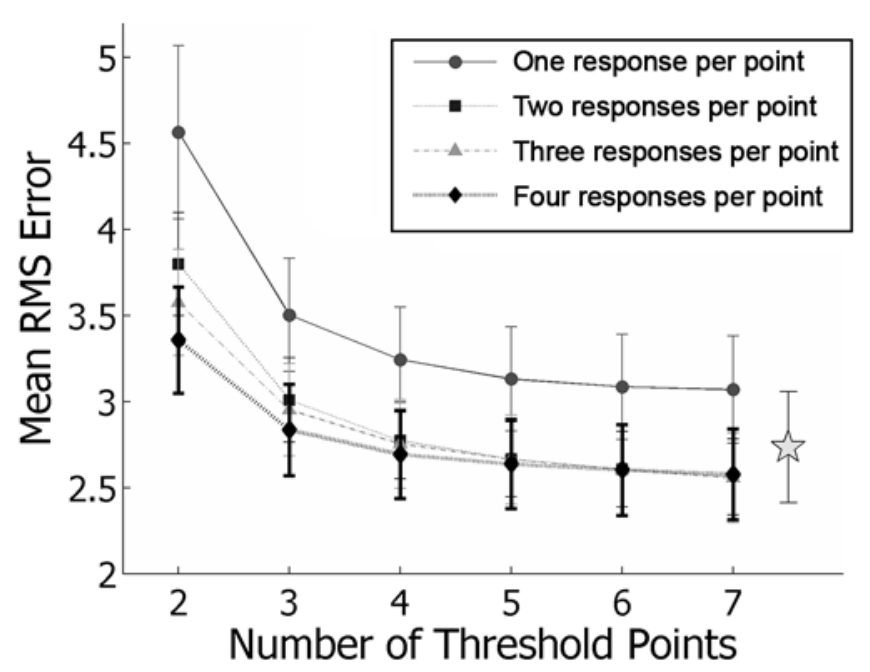

Figure 6.

Mean root mean square (RMS) error for each number of threshold points and each number of repeated responses. Error bars represent standard error. Star at lower right is mean RMS error found by using all data points collected.

Other receptors fire throughout a sensory event and with frequency coding for the magnitude of sensation [23]. Even given the differences between the motor and sensory systems, most somatosensory stimulation has been performed using the same constant pulse trains as muscle stimulation [8-9]. Recent studies have been attempting to determine a pattern of stimulation that is more effectively interpreted by the somatosensory cortex. Patterned electrical stimulation of the sacral dermatomes has been found to decrease unwanted reflex bladder contractions to a greater extent than constant stimulation [24]. In addition, experience with stimulation through nerve cuff electrodes in subjects with amputation suggests that small modulation of the amplitude or width of the pulses during stimulation trains provides a more natural sensation [12]. These methods will be investigated in future experiments to increase the occurrence of natural sensations.

Previous studies have reported chronaxie values or strength-duration time constants of sensory nerves averaging 300-650 $\mu \mathrm{s}$ using current-controlled stimulation from the skin surface [13-14]. These values are considerably higher than the 23 and $42 \mu$ s found using voltagecontrolled stimulation but consistent with the $628 \mu \mathrm{s}$ found using current-controlled stimulation in this study. Holsheimer et al. reported that the chronaxie calculated from voltage stimulation was 30 to 40 percent lower than that calculated by current stimulation using implanted deep brain stimulation electrodes [25]. In the present study, chronaxie values were 93 percent lower using voltage-controlled stimulation. The current injected using voltage-controlled stimulation is dependent on the impedance of the skin and other tissue. In the Holsheimer et al. study, electrodes were implanted closer to the target fibers and so the tissue impedance had a minimal effect. Given the significant tissue impedance when using surface stimulation, a much higher rheobase voltage was required in the present study. A higher rheobase results in a lower chronaxie, which can explain the much lower chronaxie values found here. Since the focus of this study was on finding the strength-duration threshold curve rather than the specific values, it was not necessary to find the chronaxie values that would be calculated from current-controlled stimulation.

The average rheobase voltage from the median nerve was significantly lower than from the ulnar nerve. One possible explanation for this difference is the lower impedance in the ventral skin (used for median nerve stimulation) compared to the dorsal skin (used for ulnar nerve stimulation) of the arm [26]. A second explanation involves the anatomical differences in the location of the nerve relative to the stimulating electrodes. The ulnar nerve is being stimulated where it passes through the cubital tunnel. This location reduces the excitation of tissue other than the ulnar nerve but may also require higher stimulation to penetrate the tunnel. This hypothesis will be investigated further using an anatomically based computer model.

The protocol for obtaining the strength-duration curves was modified partway through testing in response to observations that many of the trials contained data points primarily in the linear portion of the strength-duration curve, near the rheobase voltage. Calculating the duration threshold while setting the voltage amplitude higher provided data points further up the curve and may have improved the chronaxie calculation even though qualitatively the curves were still similar.

Repeated strength-duration curves from different sessions were not reliably similar. Photographs from the initial session were used to place the electrodes at the second session and to position the arm but it was impossible to ensure the identical location of the electrode relative to the nerve. The larger contributor to this disparity was likely variations in skin impedance [27-28]. The impedance of the skin to a step input has been reported at anywhere from 10 to $10,000 \mathrm{k} \Omega \mathrm{cm}^{2}$ depending on the 
electrode type and skin preparation. Skin abrasion was reported to significantly reduce the impedance [29]. In the present study, the skin was washed and cleaned with alcohol but no specific abrasion was performed. Had the skin been abraded, it may have reduced the overall rheobase voltages as well as the variability. Skin impedance has also been found to decrease after initial application of the electrodes due to electrolyte spread and penetration into the skin [26]. The present trial was designed with an initial period of stimulation without data collection to allow the subject to get used to the sensations as well as to let the impedance between the electrode and the nerve stabilize.

The location of the electrodes and the position of the arm both influenced the location of the referred sensation. If the sensation location changed during testing, subjects were instructed to adjust their position until the original sensation returned. This is a limitation of using SES and is undesirable for a clinical system. An electrode array with adjustable relative activations is being developed to allow virtual movement of the electrodes. This may also allow users to obtain sensation in multiple hand locations.

\section{CONCLUSIONS}

SES at the elbow evokes referred sensation in the hand with use of both current-controlled and voltagecontrolled stimulation. Subject-specific strength-duration curves can be generated in a timely manner and used to define the lower boundary of the effective parameter space. These curves will be used in future studies to investigate the location and type of sensation that can be evoked with the goal of obtaining a stable location and decreasing paresthesia-like sensations.

\section{ACKNOWLEDGMENTS}

\author{
Author Contributions: \\ Study concept and design: K. H. Polasek. \\ Acquisition of data: J. C. Forst, D. C. Blok, J. P. Slopsema, J. M. Boss, \\ L. A. Heyboer, C. M. Tobias. \\ Analysis and interpretation of data: K. H. Polasek, J. C. Forst, \\ D. C. Blok, J. P. Slopsema, J. M. Boss, L. A. Heyboer, C. M. Tobias. \\ Drafting of manuscript: K. H. Polasek, J. C. Forst, J. P. Slopsema. \\ Critical revision of manuscript for important intellectual content: \\ K. H. Polasek, J. C. Forst, D. C. Blok, J. P. Slopsema, J. M. Boss, \\ L. A. Heyboer, C. M. Tobias.
}

Study supervision: K. H. Polasek.

Financial Disclosures: The authors have declared that no competing interests exist.

Funding/Support: This material is the result of work supported with resources and the use of facilities at Hope College, Holland, Michigan. Additional Contributions: The authors would like to thank all of the subjects who participated in the study as well as Jill VanderStoep for assistance with the statistical analysis. All student authors were undergraduates during the study period. Currently, Johanna C. Forst, MS, is working at Medtronic in Minneapolis, Minnesota, and Carson M.

Tobias is working at Rose Medical in Grand Rapids, Michigan. Derek C. Blok is now affiliated with Wayne State University School of Medicine in Detroit, Michigan. Julia P. Slopsema is now affiliated with the Department of Biomedical Engineering at the University of Minnesota, Minneapolis, Minnesota. Lane A. Heyboer is now affiliated with the Department of Biomedical Engineering at the University of Michigan, Ann Arbor, Michigan.

Institutional Review: This study received approval from the Hope College Human Subjects Review Board. All subjects gave informed consent.

Participant Follow-Up: The authors do not plan to inform participants of the publication of this study.

\section{REFERENCES}

1. Mulvey MR, Radford HE, Fawkner HJ, Hirst L, Neumann V, Johnson MI. Transcutaneous electrical nerve stimulation for phantom pain and stump pain in adult amputees. Pain Pract. 2013;13(4):289-96. [PMID:22935086]

2. Szeto AY, Saunders FA. Electrocutaneous stimulation for sensory communication in rehabilitation engineering. IEEE Trans Biomed Eng. 1982;29(4):300-308. [PMID:7068167] http://dx.doi.org/10.1109/TBME.1982.324948

3. Buma DG, Buitenweg JR, Veltink PH. Intermittent stimulation delays adaptation to electrocutaneous sensory feedback. IEEE Trans Neural Syst Rehabil Eng. 2007;15(3): 435-41. [PMID: 17894276] http://dx.doi.org/10.1109/TNSRE.2007.903942

4. D’Alonzo M, Clemente F, Cipriani C. Vibrotactile stimulation promotes embodiment of an alien hand in amputees with phantom sensations. IEEE Trans Neural Syst Rehabil Eng. 2014;23(3):450-57. [PMID:25051556]

5. Meek SG, Jacobsen SC, Goulding PP. Extended physiologic taction: Design and evaluation of a proportional force feedback system. J Rehabil Res Dev. 1989;26(3):53-62. [PMID:2666644]

6. Fan RE, Culjat MO, King CH, Franco ML, Boryk R, Bisley JW, Dutson E, Grundfest WS. A haptic feedback system for lower-limb prostheses. IEEE Trans Neural Syst Rehabil Eng. 2008;16(3):270-77. [PMID:18586606] http://dx.doi.org/10.1109/TNSRE.2008.920075

7. Marasco PD, Kim K, Colgate JE, Peshkin MA, Kuiken TA. Robotic touch shifts perception of embodiment to a prosthesis 
in targeted reinnervation amputees. Brain. 2011;134(Pt 3): 747-58. [PMID:21252109]

http://dx.doi.org/10.1093/brain/awq361

8. Clippinger FW, Avery R, Titus BR. A sensory feedback system for an upper-limb amputation prosthesis. Bull Prosthet Res. 1974;(Fall):247-58. [PMID:4462906]

9. Dhillon GS, Horch KW. Direct neural sensory feedback and control of a prosthetic arm. IEEE Trans Neural Syst Rehabil Eng. 2005;13(4):468-72. [PMID:16425828] http://dx.doi.org/10.1109/TNSRE.2005.856072

10. Rossini PM, Micera S, Benvenuto A, Carpaneto J, Cavallo G, Citi L, Cipriani C, Denaro L, Denaro V, Di Pino G, Ferreri F, Guglielmelli E, Hoffmann KP, Raspopovic S, Rigosa J, Rossini L, Tombini M, Dario P. Double nerve intraneural interface implant on a human amputee for robotic hand control. Clin Neurophysiol. 2010;121(5):777-83. [PMID:20110193] http://dx.doi.org/10.1016/j.clinph.2010.01.001

11. Raspopovic S, Capogrosso M, Petrini FM, Bonizzato M, Rigosa J, Di Pino G, Carpaneto J, Controzzi M, Boretius T, Fernandez E, Granata G, Oddo CM, Citi L, Ciancio AL, Cipriani C, Carrozza MC, Jensen W, Guglielmelli E, Stieglitz T, Rossini PM, Micera S. Restoring natural sensory feedback in real-time bidirectional hand prostheses. Sci Transl Med. 2014;6(222):222ra19. [PMID:24500407] http://dx.doi.org/10.1126/scitranslmed.3006820

12. Tan DW, Schiefer MA, Keith MW, Anderson JR, Tyler J, Tyler DJ. A neural interface provides long-term stable natural touch perception. Sci Transl Med. 2014;6(257): 257ra138. [PMID:25298320] http://dx.doi.org/10.1126/scitranslmed.3008669

13. Mogyoros I, Kiernan MC, Burke D. Strength-duration properties of human peripheral nerve. Brain. 1996;119(Pt 2):439-47. [PMID:8800939] http://dx.doi.org/10.1093/brain/119.2.439

14. Panizza M, Nilsson J, Roth BJ, Rothwell J, Hallett M. The time constants of motor and sensory peripheral nerve fibers measured with the method of latent addition. Electroencephalogr Clin Neurophysiol. 1994;93(2):147-54. [PMID:7512921] http://dx.doi.org/10.1016/0168-5597(94)90078-7

15. Smith B, Peckham PH, Keith MW, Roscoe DD. An externally powered, multichannel, implantable stimulator for versatile control of paralyzed muscle. IEEE Trans Biomed Eng. 1987;34(7):499-508. [PMID:3610200]

http://dx.doi.org/10.1109/TBME.1987.325979

16. Smith B, Tang Z, Johnson MW, Pourmehdi S, Gazdik MM, Buckett JR, Peckham PH. An externally powered, multichannel, implantable stimulator-telemeter for control of paralyzed muscle. IEEE Trans Biomed Eng. 1998;45(4): 463-75. [PMID:9556963] http://dx.doi.org/10.1109/10.664202
17. Taylor MM, Creelman CD. PEST: Efficient estimates on probability junctions. J Acoust Soc Am. 1967;41:782.

18. Taylor MM, Forbes SM, Creelman CD. PEST reduces bias in forced choice psychophysics. J Acoust Soc Am. 1983; 74(5):1367-74. [PMID:6643848] http://dx.doi.org/10.1121/1.390161

19. Weiss G. Sur la possibilité de rendre comparables entre eux les appareils servant a l'excitation électrique. Arch Ital Biol. 1901;35:416-46. French.

20. Bostock H. The strength-duration relationship for excitation of myelinated nerve: Computed dependence on membrane parameters. J Physiol. 1983;341:59-74. [PMID:6312032] http://dx.doi.org/10.1113/jphysiol.1983.sp014792

21. Peckham PH, Knutson JS. Functional electrical stimulation for neuromuscular applications. Annu Rev Biomed Eng. 2005;7:327-60. [PMID:16004574]

http://dx.doi.org/10.1146/annurev.bioeng.6.040803.140103

22. Jenkins DB. Hollinshead's functional anatomy of the limbs and back. 8th ed. Philadelphia (PA): W.B. Saunders Company; 2002.

23. Gardner EP, Martin JH. Coding of sensory information. In: Principles of neural science. 4th ed. New York (NY): McGraw-Hill; 2000. p. 411-29.

24. McCoin JL, Bhadra N, Gustafson KJ. Electrical stimulation of sacral dermatomes can suppress aberrant urethral reflexes in felines with chronic spinal cord injury. Neurourol Urodyn. 2013;32(1):92-97. [PMID:22674730]

25. Holsheimer J, Dijkstra EA, Demeulemeester H, Nuttin B. Chronaxie calculated from current-duration and voltageduration data. J Neurosci Methods. 2000;97(1):45-50. [PMID:10771074] http://dx.doi.org/10.1016/S0165-0270(00)00163-1

26. Grimnes S. Impedance measurement of individual skin surface electrodes. Med Biol Eng Comput. 1983;21(6):750-55. [PMID:6664135] http://dx.doi.org/10.1007/BF02464038

27. Rosell J, Colominas J, Riu P, Pallas-Areny R, Webster JG. Skin impedance from $1 \mathrm{~Hz}$ to $1 \mathrm{MHz}$. IEEE Trans Biomed Eng. 1988;35(8):649-51. [PMID:3169817] http://dx.doi.org/10.1109/10.4599

28. Edelberg R. Relation of electrical properties of skin to structure and physiologic state. J Invest Dermatol. 1977; 69(3):324-27. [PMID:894073] http://dx.doi.org/10.1111/1523-1747.ep12507771

29. Huigen E, Peper A, Grimbergen CA. Investigation into the origin of the noise of surface electrodes. Med Biol Eng Comput. 2002;40(3):332-38. [PMID:12195981] http://dx.doi.org/10.1007/BF02344216

Submitted for publication May 30, 2014. Accepted in revised form March 25, 2015. 
JRRD, Volume 52, Number 4, 2015

This article and any supplementary material should be cited as follows:

Forst JC, Blok DC, Slopsema JP, Boss JM, Heyboer LA, Tobias CM, Polasek KH. Surface electrical stimulation to evoke referred sensation. J Rehabil Res Dev. 2015;52(4): 397-406.

http://dx.doi.org/10.1682/JRRD.2014.05.0128
ORCID: Katharine H. Polasek, PhD: 0000-0003-43178899

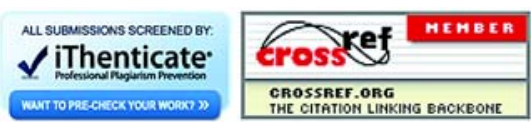

